\title{
Characterizing Benzo[a]pyrene-induced lacZ mutation spectrum in transgenic mice using next-generation sequencing
}

\author{
Marc A. Beal ${ }^{1,2}$, Rémi Gagné ${ }^{2}$, Andrew Williams², Francesco Marchetti ${ }^{2}$ and Carole L. Yauk ${ }^{2 *}$
}

\begin{abstract}
Background: The transgenic rodent mutation reporter assay provides an efficient approach to identify mutagenic agents in vivo. A major advantage of this assay is that mutant reporter transgenes can be sequenced to provide information on the mode of action of a mutagen and to identify clonally expanded mutations. However, conventional DNA sequence analysis is laborious and expensive for long transgenes, such as lacZ (3096 bp), and is not normally implemented in routine screening.

Methods: We developed a high-throughput next-generation sequencing (NGS) approach to simultaneously sequence large numbers of barcoded mutant lacZ transgenes from different animals. We collected 3872 mutants derived from the bone marrow DNA of six Muta ${ }^{\mathrm{TM}}$ Mouse males exposed to the well-established mutagen benzo[a]pyrene (BaP) and six solvent-exposed controls. Mutants within animal samples were pooled, barcoded, and then sequenced using NGS.

Results: We identified 1652 mutant sequences from 1006 independent mutations that underwent clonal expansion. This deep sequencing analysis of mutation spectrum demonstrated that BaP causes primarily guanine transversions (e.g. G:C $\rightarrow$ T:A), which is highly consistent with previous studies employing Sanger sequencing. Furthermore, we identified novel mutational hotspots in the lacZ transgene that were previously uncharacterized by Sanger sequencing. Deep sequencing also allowed for an unprecedented ability to correct for clonal expansion events, improving the sensitivity of the mutation reporter assay by $50 \%$.

Conclusion: These results demonstrate that the high-throughput nature and reduced costs offered by NGS provide a sensitive and fast approach for elucidating and comparing mutagenic mechanisms of various agents among tissues and enabling improved evaluation of genotoxins.
\end{abstract}

Keywords: Mutation spectra, Next-generation sequencing, Transgenic rodent assay, Benzo[a]pyrene, MutaTMouse

\section{Background}

Transgenic mutation reporter rodent models, such as Muta ${ }^{\text {TMM }}$ Mouse [1], enable the detection of spontaneous and induced mutations in vivo (reviewed in ref. [2]) and the identification of chemical mutagens. These transgenic rodents carry neutral reporter transgenes that can be easily recovered and detected in a bacterial host when mutated. Studies using the transgenic rodent (TGR) mutation reporter assay are very efficient and commonly used. The Organisation for Economic Co-operation and Development (OECD) has recently established guidelines for

\footnotetext{
* Correspondence: Carole.Yauk@hc-sc.gc.ca

${ }^{2}$ Environmental Health Science and Research Bureau, Healthy Environments and Consumer Safety Branch, Health Canada, Ottawa, ON K1A OK9, Canada Full list of author information is available at the end of the article
}

mutagenicity testing using this assay [3]. One major advantage of the TGR assay is that mutant transgenes can be sequenced to provide mechanistic insight into spontaneous and induced mutagenesis. DNA sequence analysis of mutations offers several major benefits in genetic toxicology: 1) mutation spectrum identifies the target nucleotides and types of mutations providing information on the mutagen's mode of action; 2) it can be used to correct for clonal expansion of mutant transgenes for a more accurate calculation of mutation frequency (versus mutant frequency); 3) it allows for a full characterization of mutational hotspots across a specific locus; 4 ) it permits a comparison of mechanisms of mutagenesis operating in different target tissues; and 5) differences in mutation spectra between treated and control groups can further 
support a mutagenic effect, especially for weak mutagens. Thus, sequence analysis provides the TGR assay with a depth of information that is not available in most genotoxicity assays.

One of the most commonly used reporter transgenes is the bacterial lac $Z$ gene because there is a simple, efficient, and robust positive selection method for identifying lacZ mutations in Escherichia coli (E. coli) [4]. Mutant detection is conducted by extracting DNA from the tissues of exposed lac Z-carrying transgenic rodents, packaging of the lac $Z$ transgene into bacteriophage and infecting host $E$. coli. Under selective conditions, only phage particles with mutated $l a c Z$ genes will be able to form plaques. Mutant frequency is calculated as the proportion of mutant plaques versus total plaques under non-selective conditions. The lac $Z$ coding sequence is the longest of the mutation reporter transgenes (3096 bp) and thus has many target nucleotide positions where mutations can induce the mutant phenotype, making it a very sensitive reporter. However, the length of the lac $Z$ gene poses challenges for sequence analysis of mutants. For this reason, few studies have sequenced the $l a c Z$ transgene to characterize mutation spectra following mutagen challenge [4-10]. Sequencing lac $Z$ requires a complementation assay to narrow down the mutation location, followed by several Sanger sequencing reactions of the section containing the mutant $[5,6,9,10]$. Therefore, the number of mutants that can be sequenced in an experiment is limited and most studies generally only sequence a few hundred mutants in total across their experimental groups. The majority of studies conducting mutation spectrum analysis use different and much shorter mutation reporter genes (cII, 294 bp; gpt, 456 bp; lacI, 1080 bp) because they are small enough to be sequenced using Sanger sequencing.

Next-generation sequencing (NGS) had been used to target and sequence pools of $c I I$ mutants from Big Blue ${ }^{\oplus}$ mice [11]. These authors pooled 150 cII mutants per sample and then sequenced 1776 mutations using 454 pyrosequencing to characterize the mutation spectra of ultraviolet radiation, 4-aminobiphenyl, and second-hand smoke, demonstrating the power of using NGS to sequence reporter genes. However, the short cII transgene is not as sensitive to induced mutations as longer genes and is therefore used much less frequently for routine screening (only $\sim 2 \%$ of studies using Big Blue ${ }^{\odot}$ or Muta ${ }^{\mathrm{Tm}}$ Mouse actually measure mutations in the $c I I$ gene [2]). With the advent of NGS, targeted sequencing of large fragments is now possible [12], and with the highthroughput nature of this technology, thousands of long fragments, such as the lac $Z$ gene, can be sequenced in a single run. Thus, NGS can be adapted to identify mutations within longer reporter genes enabling the routine incorporation of mutation spectrum analysis into the standard TGR assay.
Here we developed a high-throughput and cost-effective approach that uses NGS to characterize lacZ mutation spectra in the Muta ${ }^{\text {TM }}$ Mouse model. We collected lacZ mutants from the bone marrow of control and benzo[a]pyrene $(\mathrm{BaP})$ treated mice. $\mathrm{BaP}$ is a model mutagen known to form bulky DNA adducts with guanine bases, inducing primarily $\mathrm{G}: \mathrm{C} \rightarrow \mathrm{T}: \mathrm{A}$ transversions [6, 13-17]. Barcoding these individual mutant plaques would allow for the sequence from each plaque to be followed independently. However, this approach would not be any more efficient or cost-effective than Sanger sequencing individual mutants (Sanger: PCR amplification of multiple amplicons for sequencing vs. NGS: building a library). In the present work, we pooled mutants from each animal in a mixture, barcoded the mixture, and sequenced all the mutants in that mixture at the same time under the same barcode using an Ion Proton ${ }^{\mathrm{TM}}$ sequencer. This approach is much more efficient but does not allow us to track the mutations within a single plaque. Rather than assigning mutants to a specific plaque, mutations are identified within the pool and considered together as representative of the mouse sample. Using this approach, we enriched and sequenced an unprecedented 3872 lac Z mutant plaques from control and BaP-exposed samples in a single sequencing run and established the BaP-induced mutation spectrum with unparalleled precision. We developed an analytical pipeline to reduce false positive mutants (PCR and sequencing artefacts) that includes the use of technical replicates and stringent filtering approaches. We applied our pipeline to determine the degree of clonal expansions and make adjustments to determine mutation frequency within our population of mutants for each mouse, to precisely characterize spontaneous and BaPinduced mutation spectrum, and to determine the mutational hotspots across the lac $Z$ locus in both control and BaP-exposed mice.

\section{Methods}

\section{Animal treatment}

Six Muta ${ }^{\text {тu }}$ Mouse males (approximately 15 weeks of age; strain 40.6 carrying $29.0 \pm 4.0$ copies of lac $Z$ on chromosome 3 [18]) were exposed daily to $\mathrm{BaP}$ dissolved in olive oil by oral gavage for 28 days $(100 \mathrm{mg} / \mathrm{kg}$ body weight/day). An additional six males received olive oil and served as the vehicle controls. Three days after the last treatment, mice were anaesthetized with isofluorane and euthanized via cervical dislocation. Bone marrow was isolated from the mice by flushing the femurs with $1 \mathrm{X}$ phosphate-buffered saline. After brief centrifugation and supernatant removal, the pellet was flash-frozen in liquid nitrogen and then stored at $-80{ }^{\circ} \mathrm{C}$. All animal procedures were carried out under conditions approved by the Health Canada Ottawa Animal Care Committee. 


\section{Muta ${ }^{\mathrm{TM}}$ Mouse lacZ mutation analysis}

Bone marrow was thawed, digested in $5 \mathrm{~mL}$ of lysis buffer (10 mM Tris- $\mathrm{HCl}, \mathrm{pH}$ 7.6, $10 \mathrm{mM}$ ethylenediaminetetraacetic acid (EDTA), $100 \mathrm{mM} \mathrm{NaCl}, 1 \%$ sodium dodecyl sulfate $(w / v), 1 \mathrm{mg} / \mathrm{mL}$ Proteinase $\mathrm{K})$, and incubated at $37{ }^{\circ} \mathrm{C}$ overnight with gentle shaking. Genomic DNA was then isolated using a phenol/chloroform extraction procedure described previously $[4,5]$. DNA was dissolved in $100 \mu \mathrm{L}$ of TE buffer (10 mM Tris $\mathrm{pH} 7.6$, $1 \mathrm{mM}$ EDTA) and stored at $4{ }^{\circ} \mathrm{C}$ for several days before use. $L a c Z$ transgene mutants present in the genomic DNA were identified using the Phenyl- $\beta$-D-galactopyranoside (P-gal) positive selection assay [4]. Briefly, the $\lambda$ gt10lacZ construct was packaged into phage particles through the cohesive ends (cos sites) using the Transpack $^{\mathrm{Tm}}$ lambda packaging system (Agilent, Santa Clara, CA). Packaged phage particles were subsequently mixed with the host bacterium (E. coli lacZ $Z^{-}, g a l E^{-}, \operatorname{rec} A^{-}$, pAA119 with galT and galK) [19] in order to infect the $E$. coli with the $l a c Z$ construct. The E. coli were then plated on minimal medium containing $0.3 \%(w / v) \mathrm{P}$-gal and incubated overnight at $37^{\circ} \mathrm{C}$. P-gal is toxic to galE strains that express a functional copy of lacZ, and thus, only $E$. coli receiving a mutated copy of the lac $Z$ transcript will form plaques on the P-Gal medium. Packaged phage particles were concurrently plated on titre plates without $\mathrm{P}-\mathrm{Gal}$ to determine the total plaque-forming units (pfu). The lac $Z$ mutant frequencies were then calculated by determining the ratio of mutant pfu to total pfu. The experimental design and lac $Z$ mutation analysis used in this study followed OECD guidelines [3]. For more information on the lac $Z$ mutation assay, there is a recently published video describing the assay and procedural steps used here [20].

\section{lacZ mutant plaque collection and preparation for next-generation sequencing}

The procedure used by Besaratinia et al. [11] to amplify cII mutant plaques was adapted here for lac $Z$ mutant plaques. Figure 1 shows a brief overview of the workflow used in this experiment. After the $l a c Z$ positive selection assay was performed, lac $Z$ mutant plaques from the bone marrow of each animal were collected using a transfer pipet and pooled together into microtubes containing autoclaved milliQ sterile water $(0.3$ mutants $/ \mu \mathrm{L}$; 1 sample per tube). All of the mutants from a single animal were pooled together in a single microtube. Thus, mutations were not assigned to individual plaques; rather, all mutations within an individual mouse were identified by alignment of each read against the $\operatorname{lac} Z$ gene. For a negative control, wild-type plaques with no expected mutations were collected and processed for sequencing. Microtubes containing pooled plaques were boiled for $5 \mathrm{~min}$ and then centrifuged at 18,000 $\mathrm{g}$ for

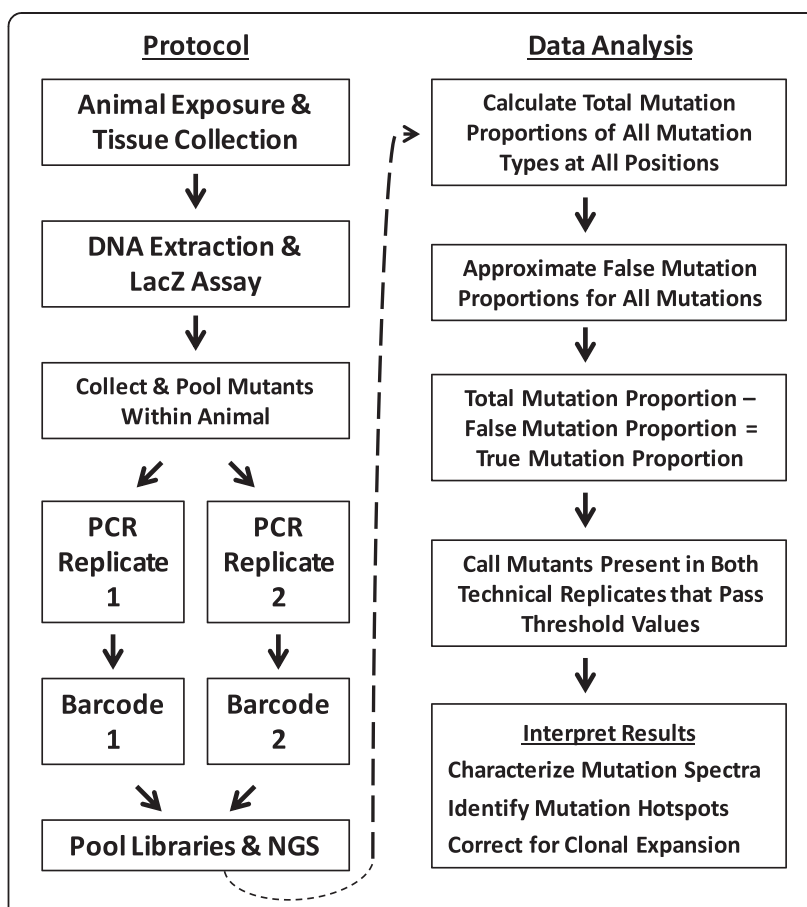

Fig. 1 Brief overview of workflow used in this study

$5 \mathrm{~min}$. The supernatant was immediately transferred to a new microtube for storage, and $10 \mu \mathrm{L}$ of the supernatant was transferred to another microtube for PCR. To control for the introduction of PCR errors, each pool of mutants was amplified in two separate reactions (i.e., two technical replicates per DNA pool). As the two replicates contain the same mutant DNA, it is expected that the same mutation should be present in both technical replicate sequences. The $30 \mu \mathrm{L}$ PCR mastermix contained a final concentration of 1X PCR buffer, 1X Q solution, 200 $\mathrm{nM}$ of each primer, $50 \mu \mathrm{M}$ dNTP, and 2.5 Units Taq DNA polymerase (Qiagen, Valencia, CA). The PCR primers used for amplification were custom designed using flanking DNA outside of the lacZ gene [GenBank:J01636.1]. The forward and reverse primers were GGCTTTACAC TTTATGCTTC and ACATAATGGATTTCCTTACG, respectively. Amplification of the lac $Z$ gene was carried out using the following thermocycle program: $95^{\circ} \mathrm{C}$ for $3 \mathrm{~min}$; 30 cycles of $95{ }^{\circ} \mathrm{C}$ for $45 \mathrm{~s}, 50{ }^{\circ} \mathrm{C}$ for $1 \mathrm{~min}, 72{ }^{\circ} \mathrm{C}$ for $4 \mathrm{~min}$; final extension at $72{ }^{\circ} \mathrm{C}$ for $7 \mathrm{~min}$. PCR products were purified using the QIAquick PCR purification kit (Qiagen) prior to library preparation.

\section{Ion Proton ${ }^{\mathrm{TM}}$ sequencing}

Next-generation sequencing of the lac $Z$ mutant products was done in-house using an Ion Proton ${ }^{\mathrm{Tm}}$ sequencer (Life Technologies, Carlsbad, CA). The Ion Xpress ${ }^{\mathrm{Tm}}$ Plus Library Kits and AB Library Builder ${ }^{\mathrm{TM}}$ (Ion Xpress Plus Library Protocol v. 1.00) were used to prepare one library for each animal's pool of mutants from purified PCR 
products (for a total of 24 libraries). The procedure outlined in the user's manual was followed with the exception of a 10 min custom shearing time and the replacement of Agencourt ${ }^{\circ}$ AMPure $^{\circ}$ XP Reagent with SPRIselect ${ }^{\circ}$ Reagent (Beckman Coulter, Brea, CA). The P1 Adapter and barcoded A adapters provided in the Ion Xpress ${ }^{\text {Tw }}$ Barcode Adapter kits were ligated to the ends of the fragmented libraries (i.e., each PCR product received a unique barcode). Gel electrophoresis of the libraries was performed on $\mathrm{E}_{-\mathrm{Gel}^{\circ}}$ SizeSelect $^{\mathrm{Tm}} 2 \%$ Agarose Gel using the $\mathrm{E}-\mathrm{Gel}^{\circ}$ iBase $^{\mathrm{Tu}}$ and visualized using the E-Gel ${ }^{\circ}$ Safe Imager ${ }^{\text {tw }}$ transilluminator. Fragments that were between $\sim 175$ and $\sim 225$ bp were excised from the gel and purified using the Invitrogen ${ }^{\mathrm{m}}$ PureLink $^{\circ}$ Quick Gel Extraction Kit. Libraries were then amplified using the Library Amplification Primer Mix (Ion Plus Fragment Library Adapters kit) and Platinum ${ }^{\circ}$ PCR SuperMix High Fidelity. Each amplified library was quantified/ qualified using the Agilent ${ }^{\circ}$ High Sensitivity DNA Kit and Agilent ${ }^{\circ}$ Bioanalyzer 2100 Instrument. Aliquots of each library were pooled together for a total final concentration of $16 \mathrm{pM}$. Emulsion PCR (em-PCR) of the pooled libraries was carried out using Ion Sphere ${ }^{\mathrm{Tm}}$ particles on the Ion OneTouch ${ }^{\text {Tx }} 2$ system. The Ion OneTouch ${ }^{\text {Tx }}$ ES was used to enrich for particles containing em-PCR products and the Qubit $^{\circ}$ 2.0 Fluorometer was used to assess the quality of enrichment. Enriched particles were deposited into the wells of an Ion $\mathrm{P} 1^{\mathrm{Tx}}$ chip (version 1) and sequenced by semi-conductor sequencing [21].

\section{Bioinformatics}

The Proton $^{\mathrm{Tu}}$ Torrent Server version 3.6.2 interpreted the sequencing data and generated FASTQ files for each barcoded library. Reads were trimmed to remove low quality base calls and aligned to the reference sequence [GenBank: J01636.1] using Bowtie2 [22] (Settings: "- local" mode with "- very-sensitive-local"). The lacZ gene in our Muta ${ }^{\text {mi }}$ Mouse colony has four variations [6] relative to the reference coding sequence [GenBank:V00296.1] [23] that were corrected for.

Following alignment, a pileup format was prepared for each sample using SAMtools [24]. From the sample pileups, the number of reads that were wild-type or polymorphic (base substitutions/indels) was determined for each nucleotide position of the lac $Z$ gene. At each nucleotide position, a proportion of reads is expected to carry a technical artefact (e.g., PCR artefacts and sequencing noise) for each possible mutation type (referred to as the false mutation proportion: see glossary in Table 1). However, these false mutation calls are consistent across samples (Additional file 1: Figure S1) and thus the false mutation proportion of each mutation type can be estimated individually at each nucleotide position. In order to prevent outlier libraries from influencing the background
Table 1 Glossary

Mutant - A plaque, grown on P-Gal media, that contains a loss of function mutation in the lac $Z$ transgene.

Mutation - The change in DNA sequence (point mutation, indel) that knocks out the function of the lac $Z$ transgene. There may also be silent mutations that can co-occur on the same lacZ copy as a loss of function mutation ("hitchhiker mutations").

Clonal Expansion - When a cell containing a mutation produces daughter cells containing that same mutation, propagating it throughout the tissue.

False Mutation Proportion - At each nucleotide position, a proportion of the reads may contain a technical artefact and this proportion is consistent across samples. For example, if at position 25 , the median frequency of $G \rightarrow A$ substitutions across all samples was one in every thousand reads, the false mutation proportion would be 0.001 .

Total Mutation Proportion - At each nucleotide position, this is the mutation proportion for each mutation type within a single sample. For example, if sample 3 had a $G \rightarrow$ A substitutions at position 25 in $0.5 \%$ of reads, the total mutation proportion would be 0.005 .

True Mutation Proportion - The true mutation proportion of each mutation type at each position is determined by subtracting the false mutation proportion from the total mutation proportion. In the above example the true mutation proportion would be 0.004 . This value is used to determine mutation counts.

Independent Mutations - Mutations that appear multiple times in a same sample due to clonal expansion are only counted as one mutation per sample. For example, if sample 1 had five $G \rightarrow A$ substitutions at position 25 , this would be called a single independent mutation at position 25.

Recurrent Mutations - If a mutation occurs more than once within a sample it is considered recurrent as is likely the result of clonal expansion. In the previous example there are five $\mathrm{G} \rightarrow \mathrm{A}$ recurrent mutations at position 25 .

reading, false mutation proportions were estimated by calculating the median rate of occurrence of each mutation type across all samples for each nucleotide position. The data were then normalized by subtracting the false mutation proportion from the total mutation proportion to determine the true mutation proportion at each nucleotide position (Additional file 1: Figure S2; see glossary for clarification). Due to homopolymer sequencing errors, the false mutation proportion for some insertions/deletions (indels) can be much higher than false mutation proportions for base substitutions. To minimize false indel calls, indels appearing with a false mutation proportion higher than the highest false mutation proportion for base substitution calls were ignored.

Variants that remained following the false mutation proportion subtraction were considered mutations if the true mutation proportion was above threshold values determined based on the number of mutant plaques recovered from each sample and used in the sequencing (Additional file 1: Table S1). For example, if a mutant pool for an individual animal consisted of 100 plaques, a single mutation should be present in approximately $1 \%$ of the sequenced reads ( $1 \%$ of reads would have the variant while $99 \%$ would have the reference nucleotide). 
To determine the threshold that should be set for identifying true mutations, the data were first analyzed using three different thresholds to optimize the sensitivity of the assay. The thresholds were set at: 1) the exact proportion for the mutation expected; 2) $75 \%$ of the expected proportion; and 3) $50 \%$ of the expected proportion. Using the previous example, the thresholds would be $0.01,0.0075$, and 0.005 , respectively. In addition, in order for a mutation to be counted it needed to be present at values above the threshold in both technical replicates.

The average between the true mutation proportions of each technical replicate was used to provide a rough estimation of the total number of each type of mutation. For example, a base substitution present at a frequency of 0.10 would be counted as ten mutations in a sample that had a minimum threshold of 0.01 . These values were used to determine the distribution of mutations across the lac $Z$ gene for both the BaP-treated and control groups and identify mutation hotspots. In contrast, only independent mutations were used to determine the lacZ mutation spectra for both groups.

Finally, we examined the sequence sites of each specific mutation identified in the lac $Z$ gene. The DNA codon table was used to determine whether base substitution changes observed in the coding sequence would result in amino acid changes in the protein. Based on this analysis, mutations were classified as nonsense, missense or silent.

\section{Statistical analysis}

Statistical analyses were performed using $\mathrm{R}$ programming language to compare mutant frequencies and mutation spectra between control and treated animals [25]. The lac $Z$ mutant frequencies in the bone marrow of control and BaP-treated animals were compared by Poisson Regression. Statistical comparisons of mutation types between control and treated groups were done using Pearson's chi-squared test of independence.

Mutations that occurred more than once in the same animal were considered to be derived from clonal expansion (see glossary) from a single 'independent' event. The formula of [(total mutations - independent mutations)/total mutations] was used to estimate clonality. To test the method's ability to estimate clonality, eight mutant plaques from different animals (each mutation identified by Sanger sequencing) were subcloned and then mixed together in different proportions (Additional file 1: Table S2). One of these mixtures contained different sized plaques to see whether plaque size affected clonality estimation. As an additional control, 100 random mutants from 100 different animals were pooled together to estimate the ability to identify unique mutations that were not expected to be clonally amplified.
The observed proportions recovered by sequencing were then compared with the expected proportions of each mutant mixture. A Fitting Linear Models algorithm $[26,27]$ in $\mathrm{R}$ was used to estimate the limit of detection (LOD) for accurately quantifying mutant count using NGS. The algorithm searches for the LOD with the lowest squared error using 1000 iterations with decreasing step size. The model estimated that the LOD was four mutants (horizontal blue line in Fig. 2) and thus mutants with an observed count of four or less were set to a count of one. Above the LOD, the relationship between expected and observed mutant count is linear $(P=$ 0.4249). Thus, using the LOD/Linear model we adjusted the count of each mutant in the $\mathrm{BaP}$ experiment for clonality measurements with the formula: Adjusted Mutant Count $=($ Observed Count - Intercept $) /$ Slope for mutant counts $>4$ where the $y$ intercept $=0.3123$ and the slope $=1.1363$. Clonality was then calculated based on the adjusted mutant counts. For example, a count of three mutations was adjusted to one mutation and a count of 50 mutations was adjusted to 44 mutations.

\section{Results}

\section{lacZ mutant frequency}

As expected, bone marrow lac $Z$ mutant frequencies were significantly higher in BaP-treated mice (701.7 \pm $\left.49.3 \times 10^{-5}\right)$ than control mice $\left(8.6 \pm 2.0 \times 10^{-5}\right) \quad(\mathrm{P}<$ $0.0001)$. This increase in mutant frequency is consistent with previous results [28]. However, the spontaneous mutant frequency was higher than expected based on historical controls (example: [28]), mostly due to animal Control20, which had a mutant frequency of $17.8 \times 10^{-5}$. This animal was included in the study to test the method's ability to detect clonal expansion.

\section{Sequencing output and read coverage}

Sequencing of the pooled libraries using one Ion $\mathrm{P} 1^{\mathrm{mm}}$ chip (version 1) gave 87,666,405 reads of $95 \mathrm{bp}$ mean length for a total of $8.3 \mathrm{~Gb}$. The number of usable reads was reduced to $66 \%$ after removal of polyclonal Ion Sphere $^{\mathrm{rm}}$ particles $(29 \%)$ obtained from the pooled library. Thus, the average nucleotide coverage per library was $9.1 \pm 0.4 \times 10^{4}$. The starts and ends of the reads across the gene appeared to be uniform (Additional file 1: Figure S3).

\section{False mutation proportion subtraction and threshold comparisons}

An analysis of our data using conventional approaches revealed that most existing algorithms or variant callers were not equipped to detect rare mutations in pooled samples. Thus, we explored the use of a false mutation proportion subtraction methodology that we developed in-house. False mutation proportion refers to the sequencing artefacts 


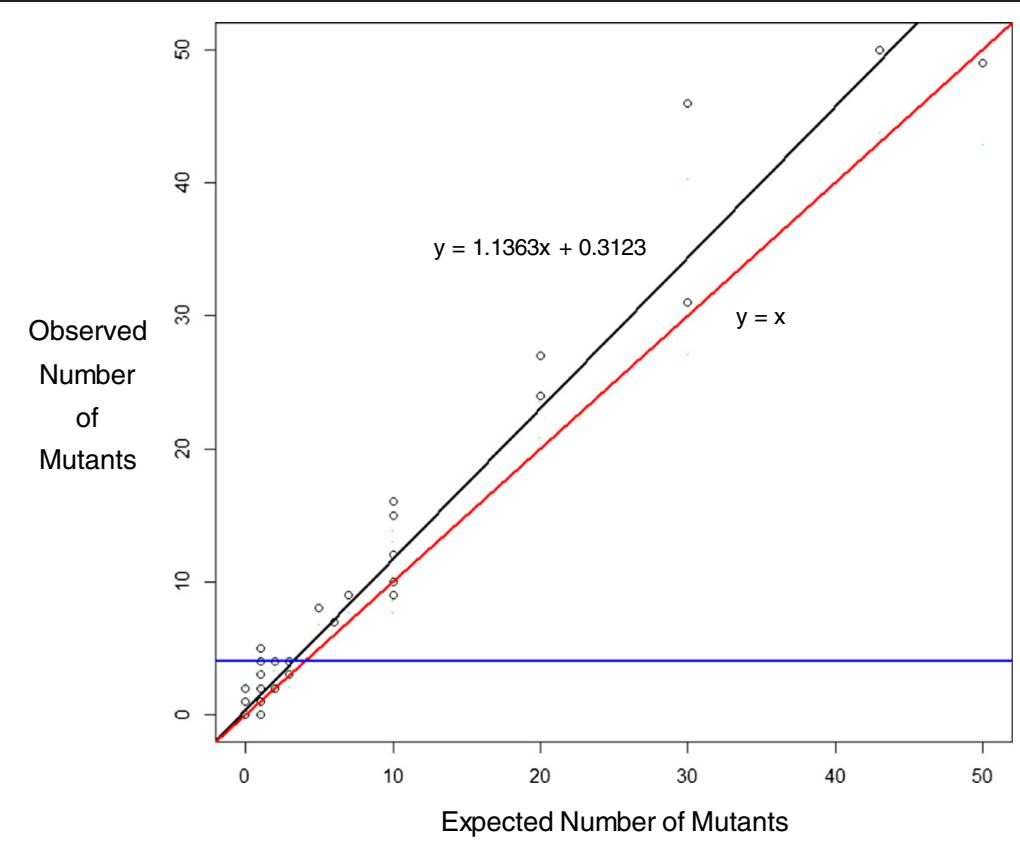

Fig. 2 Correlation between observed and expected mutant counts. The red line shows a 1:1 ratio and the black line shows the true relationship between expected and observed mutant count. The plot shows that mutants with low counts cannot be accurately quantified. This results in a non-linear relationship between expected and observed mutant count $(P<0.0001)$. Hence, a limit of detection/linear model was used to adjust the total mutation counts of each independent mutation

that occur by chance at each nucleotide position. We use the term 'proportion' here to differentiate the proportion of reads carrying errors (background noise) from reads with true mutations. The false mutation proportions (see glossary for specific definition) for base substitutions ranged from $1.70 \times 10^{-5}$ to $2.15 \times 10^{-2}$ (median: $4.25 \times 10^{-3}$ ), and from $1.64 \times 10^{-5}$ to $4.64 \times 10^{-1}$ (median: $7.52 \times 10^{-3}$ ) for indels (Additional file 1: Figure S4). As the number of indel technical errors increase, the ability to reliably call mutations diminishes. Thus, indels were only called if the false mutation proportion was less than $2.15 \times 10^{-2}$. Indels occurring at sites with a false mutation proportion greater than this were not included in the analysis. In the final analysis, this resulted in the removal of 21 out of 65 insertions (3/6 control, 18/59 BaP) and 99 out of 297 deletions (31/53 control, 68/244 BaP).

After correcting for the false mutation proportion, we explored mutation calling using three different thresholds for optimization (Additional file 1: Table S1). With the stringent threshold (i.e., the exact proportion expected), 152 and 854 independent mutations were recovered in both technical replicates for the control and $\mathrm{BaP}$ groups respectively (Table 2 ). The total number of mutations (unadjusted by LOD/Linear model) recovered out of 872 and 3000 mutant plaques from control and BaPtreated samples were 583 (67 \%) and 2077 (69 \%), respectively. Applying the two less stringent thresholds increased the number of mutations called from 2660 to
2912 and 3282, respectively; however, the discrepancies between technical replicates greatly increased. Thus, all downstream analyses were done on mutations called using the most stringent threshold. As expected, applying the most stringent threshold resulted in no mutation calls for the wild-type plaques.

\section{Detection of clonal expansion}

We created five control libraries (Additional file 1: Table S2) in which the number of mutations was known in order to determine the ability of our NGS approach to accurately predict the number of copies of each mutation. In addition, a library of 100 random mutants was sequenced to determine our ability to detect unique mutations. For each mutation detected, we divided the true mutation proportion by the expected mutation proportion for a single mutation (threshold) to estimate the number of copies of that mutation in any given sample. In the library of 100 random mutants there were 49 independent mutants detected with a total count of 102 recurrent mutations, which is a higher proportion of recurrent mutations than was expected [5]. It is possible that some of the recurrent mutations occurred at mutation hotspots. For example, five independent mutants and 15 recurrent mutations were detected at four of five known mutation hotspots [8]. However, it is more likely that some mutants were not detected while the count of the detected mutants was overestimated. When the expected mutant 
Table 2 Summary of all mutations called using three different thresholds

\begin{tabular}{|c|c|c|c|c|c|c|c|c|c|c|}
\hline & \multirow[b]{2}{*}{ Threshold } & \multicolumn{3}{|c|}{ Independent Mutations } & \multicolumn{3}{|c|}{ Total Mutations } & \multicolumn{3}{|c|}{${ }^{a}$ Mutants Removed } \\
\hline & & ${ }^{\mathrm{b}}$ Base Subs. & ${ }^{c}$ Ins & ${ }^{\mathrm{d}}$ Dels & Base Subs. & Ins & Dels & Base Subs. & Ins & Dels \\
\hline \multirow[t]{3}{*}{ Control } & Stringent & 127 & 3 & 22 & 542 & 7 & 34 & 14 & 101 & 117 \\
\hline & Medium & 159 & 7 & 27 & 574 & 7 & 39 & 18 & 193 & 223 \\
\hline & Low & 190 & 12 & 36 & 605 & 16 & 48 & 55 & 426 & 420 \\
\hline \multirow[t]{3}{*}{$\mathrm{BaP}$} & Stringent & 637 & 41 & 176 & 1583 & 66 & 428 & 108 & 750 & 577 \\
\hline & Medium & 767 & 60 & 239 & 1714 & 85 & 493 & 183 & 1139 & 818 \\
\hline & Low & 921 & 125 & 338 & 1869 & 151 & 593 & 374 & 1846 & 1284 \\
\hline
\end{tabular}

${ }^{a}$ Mutants removed refer to the number of variants that passed the threshold in one technical replicate but not the other

${ }^{\mathrm{b}}$ Base substitutions

Insertions

${ }^{\mathrm{d}}$ Deletions

count was compared with the observed mutant count in the five control libraries (Fig. 2), we found that the count of each mutant was overestimated. Therefore, a limit of detection/linear model was developed to adjust the recurrent mutation counts of each independent mutation. Based on the model, the total mutation count for the library containing 100 random plaques was estimated to be 55 mutations. The mutant count estimation for the experimental libraries was 1652 mutant sequences (411 control, $1241 \mathrm{BaP}$ ) from 1006 independent mutations. A total of 917 mutants in the experimental libraries fell below the LOD, indicating a relatively small proportion of mutants underwent clonal expansion. The adjusted average clonality in the control and $\mathrm{BaP}$ samples was 43 and $31 \%$ respectively (Additional file 1: Table S3), which is comparable to what was measured at the $c I I$ locus in the same mice (O'Brien, unpublished) and in other studies [2]. After adjusting for clonality, the $l a c Z$ mutation frequencies were $4.0 \pm 1.0 \times 10^{-5}$ and $484.0 \pm 34.9 \times 10^{-5}$ for control and $\mathrm{BaP}$ mice, respectively. The clonality adjustment revealed that the raw control mutant frequency was elevated in part by the presence of one outlier animal with extensive clonal expansion (86\%). After correcting for clonal expansion, the mutation frequency of this animal was in line with the mutation frequency of the rest of the animals (within one standard deviation of the mean; Additional file 1: Table S3). The model for adjusting clonality showed that $\mathrm{BaP}$ induction was 121-fold higher than control.

\section{lacZ mutation spectra and distribution}

The BaP mutation spectrum in bone marrow had significant differences from the control spectrum (Fig. 3). The proportions of transitions, transversions, and indels were $0.35,0.49$, and 0.16 in the control and $0.14,0.61$, and 0.25 in the $\mathrm{BaP}$ animals, respectively. The proportion of $\mathrm{G}: \mathrm{C} \rightarrow \mathrm{T}: \mathrm{A}$ and $\mathrm{G}: \mathrm{C} \rightarrow \mathrm{C}: \mathrm{G}$ transversions was significantly increased in BaP-treated animals relative to controls $(P<0.05)$. In addition, the proportion of $\mathrm{G}: \mathrm{C} \rightarrow$ A:T transitions was reduced $(P<0.0001)$ following $\mathrm{BaP}$ treatment. These findings are consistent with the lacI mutation spectrum in BaP-treated mouse liver [29] (Additional file 1: Figure S5). There were no significant differences in the proportion of the other mutation types between control and BaP-treated groups. When comparing the absolute frequencies of each mutation type, BaP exposure increased all mutation types above control except for A: $\mathrm{T} \rightarrow \mathrm{G}: \mathrm{C}$ transitions (Additional file 1: Figure S6). To further test the accuracy of our NGS data, we compared the control and $\mathrm{BaP}$-induced bone marrow lac $Z$ mutation spectrum with published mutation spectra for transgene reporters from control and BaP-treated mice. The comparisons of control (Additional file 1: Figure S7) and BaP-induced (Additional file 1: Figure S8) spectra were consistent with the published data using Sanger sequencing, demonstrating that our NGS approach accurately identifies mutation spectra.

Plotting the locations of the independent (Fig. 4) and recurrent (Additional file 1: Figure S9) base substitutions along the lac $Z$ transgene to identify mutation hotspots demonstrated that the majority of induced and spontaneous mutations were at $\mathrm{G}: \mathrm{C}$ base pairs (Additional file 1: Table S4). Within the lac $Z$ transgene there are 12 short $\mathrm{CpG}$ sequences $\geq 5$ nucleotides long. At these $\mathrm{CpG}$ sites there were five control point mutations (1G:C $\rightarrow$ A:T, 3G:C $\rightarrow$ T:A, 1G:C $\rightarrow$ C:G) and 36 BaP point mutations $(1 \mathrm{G}: \mathrm{C} \rightarrow \mathrm{A}: \mathrm{T}, 24 \mathrm{G}: \mathrm{C} \rightarrow \mathrm{T}: \mathrm{A}, 11 \mathrm{G}: \mathrm{C} \rightarrow \mathrm{C}: \mathrm{G})$. All nucleotide positions that had more than two independent mutations were at $\mathrm{G}: \mathrm{C}$ sites in both control and $\mathrm{BaP}$ groups. Previously identified mutation hotspots in the lacZ gene using Sanger sequencing, based on $\sim 500$ mutations [5-10], were also hotspots in our dataset (Additional file 1: Figure S10). In all previous studies, there were 67 nucleotide positions with more than one mutation, and 51 (76\%) of these positions had a mutation in our dataset. We found mutations at all of the nucleotide positions that had more than four mutations across the previously published mutation hotspots. In the NGS data, there were 29 hotspots with more than four independent mutations across the different animals; 


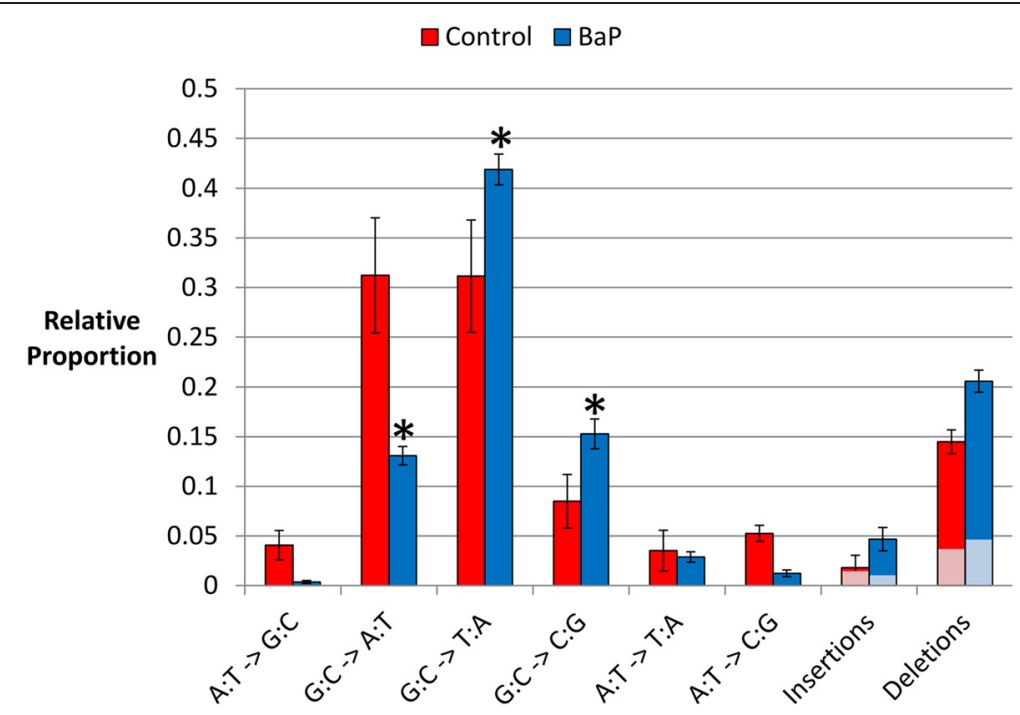

Fig. 3 Relative proportion of control and BaP-induced lacZ mutations in bone marrow. Only independent mutations were considered. Mutation types with asterisks are significantly different between control and treated groups. The light-colored boxes within indels represent the fraction of indels that was inside or next to homopolymer stretches $\geq 3$ bp long. The error bars represent the standard error between samples

of these nucleotide positions only two (7\%) were not identified using Sanger sequencing in previous studies.

The majority of indels that we observed were gains/ losses of single nucleotides. There were 34 indels (of 242 in total) that involved two nucleotides: $32 / 198$ deletions (3/22 control, 29/176 BaP); and 2/44 insertions (0/3 control, 2/41 BaP). Detecting indels was not as reliable as detecting base substitutions due to difficulty in sequencing homopolymer regions; sequencing accuracy of homopolymers falls to $97 \%$ at homopolymer stretches of five or more base pairs [21]. In the lacZ gene, there are 591 short homopolymers (462 two mononucleotide repeats; 97 three mononucleotide repeats; and $32 \geq 4$ mononucleotide repeats). This means that $1353 \mathrm{bp}$ (44\%) of the lacZ gene are within a short homopolymer.
To test for a homopolymer bias, indels were plotted across the lacZ gene (Fig. 5, Additional file 1: Figure S11). The numbers of mutations occurring inside or adjacent to homopolymer stretches $\geq 3$ bp long were: 2 out of 3 control insertions, 5 out 22 control deletions, 7 out of $41 \mathrm{BaP}$ insertions, and 33 out of $176 \mathrm{BaP}$ deletions (Fig. 3). Furthermore, only ten (31\%) of the 32 longest homopolymers in the lacZ gene had mutations within or adjacent to the homopolymers. Thus, the false mutation proportion subtraction accounted for many sequencing artefacts occurring around homopolymer regions.

Using the coding sequence of lacZ, we identified the amino acid changes that would result from each mutation. This analysis revealed that the proportion of missense, nonsense, and silent mutations was 39, 59, and

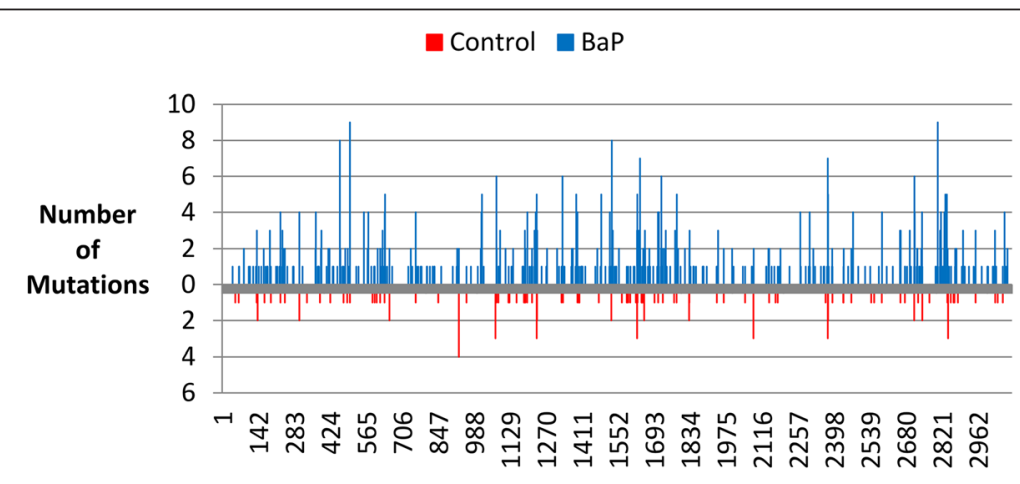

Nucleotide Position

Fig. 4 Distribution of unique base substitutions across the lac $Z$ transgene for control and BaP samples. BaP base substitutions are on the positive $y$-axis and control substitutions are on the negative $y$-axis 
- Control Insertions $\square$ Control Deletions $\square$ BaP Insertions $\square$ BaP Deletions

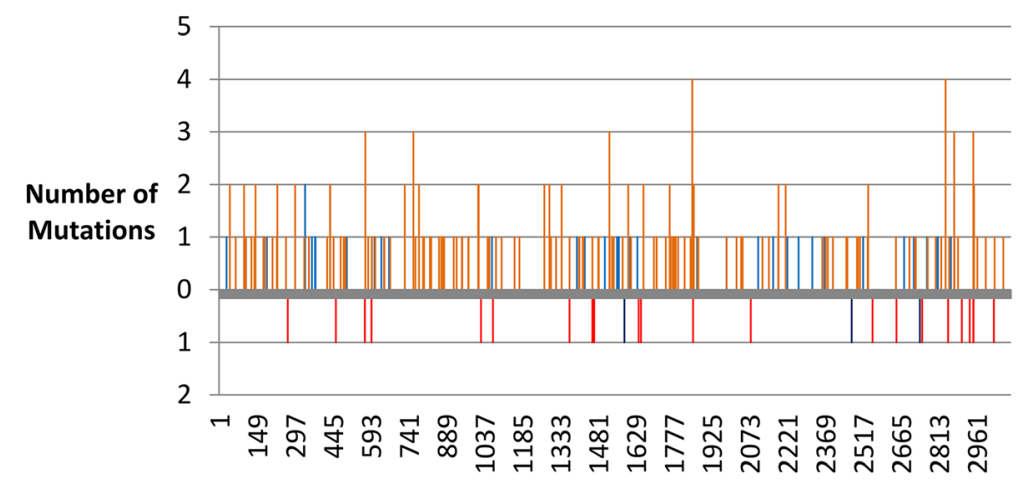

Nucleotide Position

Fig. 5 Distribution of unique indels across the lacZ transgene for control and BaP samples. BaP indels are on the positive $y$-axis and control indels are on the negative $y$-axis

$2 \%$ in the control, and 50, 44, and $7 \%$ in the BaP group, respectively. The silent mutations that were identified by NGS are likely "hitchhiker" mutations that occurred in the same plaque as a phenotype-inducing mutation. Sanger sequencing of mutant plaques shows that the proportion of plaques with more than one mutation (synonymous or non-synonymous) can be as high as $11 \%$, and the proportion of plaques with mutations separated by hundreds of nucleotides is around $3 \%$ [6]. Also, the proportion of silent mutations observed here is consistent with the proportion of silent mutations detected using Sanger sequencing $(<6 \%)$ [5, 6, 8-10, 29]. The number of missense, nonsense, and silent mutations that occurred more than once at a nucleotide position was 10,7 , and $0(20,9$, and $0 \%)$ for the control, and 55 , 62 , and $4(18,22$, and $8 \%)$ for the BaP, respectively. Taking both groups into account there were 66,81 , and 4 positions where there was more than one missense, nonsense, and silent mutation, respectively. The low number of recurring silent mutations is as expected and reinforces the reliability of the data.

We also performed simulations, using random mutant sampling, to determine the minimum number of mutants per samples that would be sufficient to achieve a consistent mutation spectrum in the $\mathrm{BaP}$ group. In this simulation, mutations were sampled from 375, 348, 364, 360, and $299 \mathrm{BaP}$ mutants $(150,142,161,138$, and 139 $\mathrm{BaP}$ mutations) in five of the six treated animals, respectively. One animal had to be excluded because some of the mutation types did not occur frequently enough for accurate simulations. There were no significant differences in the mutation spectra of the five remaining animals $(P=0.3)$. As shown in Additional file 1: Figure S12, the results of our simulations demonstrated that sequencing approximately 100 mutants from a mouse provides an $80 \%$ power to obtain the true mutation spectrum for that individual $(P<0.05)$.

\section{Discussion}

We developed a simple, cost-effective, and high-throughput method to characterize the mutation spectrum of a transgenic mutation reporter locus in rodents using targeted NGS. This method can be adapted for any mutation reporter model using any NGS platform. In addition, the data analysis is simple and non-subjective. Using our conservative approach, we identified 1006 independent mutations within the lac $Z$ transgene allowing us to characterize the spontaneous and BaP-induced mutation spectra with unparalleled precision. As expected, BaP exposure induced primarily G:C $\rightarrow$ T:A transversions. Our approach also allowed us to identify 1652 total mutations (including recurrent mutations that occurred more than once per mouse), permitting clonal expansion correction and making the assay $50 \%$ more sensitive in detecting increases in mutation frequencies. Furthermore, comparisons of our NGS results with previous results obtained using Sanger sequencing show that the mutation spectra (Fig. 3), mutation hotspots (Additional file 1: Figure S10), and amino acid changes were as expected, demonstrating the robustness of our approach.

Characterization of the mutation spectra is important for identifying which sites are targeted by chemicals to elucidate mutagenic mechanism (e.g., what types of adducts are formed, faulty DNA repair processes, etc.). We investigated the mutation signature of $\mathrm{BaP}$ because it is a model carcinogen and its metabolic activation is well understood [30, 31]. BaP is metabolized by cytochrome P450 enzymes and epoxide hydrolase to mutagenic metabolites including benzo[a]pyrene-7,8-dihydrodiol-9,10epoxide (BPDE) [31-33]. BPDE forms DNA adducts with 
the 2-amino group (N2) of guanine, and to a lesser extent with $\mathrm{N} 6$ and $\mathrm{N} 4$ of adenine and cytosine, respectively [32-34]. BPDE also produces reactive oxygen species through the spontaneous rearrangement of the epoxides or redox cycling, which also react with DNA leading to oxidative DNA damage, such as 8-oxo-deoxyguanosine [32]. These BaP damaging events explain the high proportion of guanine base substitutions, specifically $G \rightarrow T$ and $\mathrm{G} \rightarrow \mathrm{C}$, seen here in bone marrow. In total, $89 \%$ of BaPinduced mutations occurred at $\mathrm{G}: \mathrm{C}$ nucleotides and all of the BaP-associated mutation hotspots were at G:C nucleotides. However, the refined mutation spectra obtained from sequencing thousands of mutants demonstrated that absolute mutation frequencies of all mutation types were elevated relative to the control with the exception of $\mathrm{A}: \mathrm{T} \rightarrow \mathrm{G}: \mathrm{C}$ transitions. Thus, BaP is a potent mutagen capable of inducing transversions at any nucleotide and transitions at $\mathrm{G}: \mathrm{C}$ nucleotides.

One of the main reasons for sequencing mutants is to account for clonal expansion [3]. This can cause a tissue sample to have a higher mutant frequency than the rest of the samples within a group and falsely over-represents independent mutation events. To correct for clonal expansion, a subset of the mutant plaques from the tissue sample is sequenced to identify recurrent mutations arising from one progenitor cell that may be inflating the mutant frequency. Recurrent mutations are only counted as one mutation and the mutant frequency is adjusted accordingly (to reflect the true mutation frequency). Plotting the mutation distribution of all animals revealed potential clonal expansion events. Using the LOD/Linear model the corrected clonality estimates were 43 and $31 \%$ in the control and exposed groups respectively. Correcting for clonality revealed that the fold increase in mutation frequency following BaP treatment was $\sim 120$-fold. This suggests that although NGS may overestimate the clonality of a tissue sample, the overestimation is consistent across samples and therefore a relative clonality comparison between groups can be achieved.

It should also be noted that the number of mutants sequenced can influence the clonal expansion estimation. In this study we sequenced a large number of mutants unmatched by any other study, and for this reason we may be seeing higher levels of clonality. Simulations show that had only 25 mutations been sequenced per animal (more typical of past experiments using Sanger sequencing [35]), the clonality would have remained at $43 \%$ in the control group, but would have been reduced to $9 \%$ in treated samples. The differences in clonality between actual and simulated measurements highlight the need to use caution when comparing clonality results determined by sequencing large versus small numbers of mutants. The lower clonality observed in the treated group can be explained by the presence of thousands of BaP-induced mutations that are reducing the chance of randomly sampling spontaneous mutations that were clonally expanded during mouse development.

The need to account for clonality was clearly demonstrated by the presence of one outlier control animal with a high mutant frequency in our study (Additional file 1: Table S3). Because of this outlier, which had $86 \%$ clonality, our control group had a mean mutant frequency that was higher than expected based on previous studies [2]. However, after correcting for clonal expansion there was less variability in mutation frequencies within each group. Furthermore, the adjusted spontaneous mutation frequency was consistent with historical values for bone marrow from our laboratory [28, 36]. Finally, correcting for clonal expansion demonstrated that the mutation induction caused by $\mathrm{BaP}$ was underestimated by $50 \%$ in the standard non-sequenced based approach. Had a weaker mutagen been tested, the transgenic rodent assay may have given a false-negative result without adjustment of clonal expansion. These examples show the added value of sequencing to measure clonality when interpreting the results of the TGR assay and demonstrate that our method can be used to correct for clonal expansion events at the lac $Z$ locus to improve the reliability of reporter gene data.

There are notable strengths in our approach. First, our method uses a simple false mutation proportion subtraction to minimize sequencing artefacts and alignment errors. It is known that different NGS platforms and alignment tools have biases that can introduce false positives [37-40]. For example, the accuracy of semiconductor sequencing decreases with increased homopolymer length [21], as is seen with pyrosequencing [41, 42]. However, these biases are consistent between the samples at each nucleotide position (Additional file 1: Figure S1) and thus can be corrected (Additional file 1: Figure S2). After applying the correction, we were able to identify independent mutations along the lac $Z$ gene and approximate the number of times each mutation occurred. Second, we used technical replicates (two PCR amplifications) for each sample as a strategy to minimize the number of false-positives. This was done to account for errors that occurred during PCR as a result of Taq replication errors or thermal DNA damage [43, 44]. Using technical replicates does not reduce the throughput of the sequencing, and only marginally increases the cost of the experiment. Although this approach is very conservative and reduces the number of mutations called (i.e., we identified 1652 mutants from 3872 plaques), it is currently needed to eliminate false-positives, especially for indels, and accurately call mutations (Table 2). Thus, we strongly recommend that technical replicates be used with this method to control for PCR-based errors, allowing for 
accurate characterization of mutation spectra. Given the significant increases in the number of mutants that can be sequenced relative to Sanger sequencing, our stringent criteria for calling mutations does not reduce the power of the approach; indeed, we have sequenced an unprecedented number of lac $Z$ mutations within a single experiment. This method was designed to be modular; therefore, researchers can explore the use of other bioinformatics and statistical tools that best fit their data. Before setting up this protocol in a laboratory, it is recommended that experiments be conducted using positive and negative controls to optimize parameters as we have done here.

The number of mutants that should be pooled per barcode must be considered carefully in the experimental design. As the number of mutants increase, the threshold to call a mutation decreases and the chance of identifying an artefact as a mutation increases. In addition, having too many mutants pooled per barcode will decrease the detection sensitivity of the NGS method. Contrarily, having too few mutants will not provide an accurate mutation spectrum. In order to approximate the number of mutants that should be pooled per barcode, we performed multiple data simulations based on the available results. These simulations show that sampling around 100 mutants per animal is sufficient to achieve reliable mutation spectra with a power of $80 \%$, and therefore, pooling approximately 170 mutants per barcode (assuming a mutant recovery of $\sim 60 \%$ ) should be more than enough for any sequencing application. If sequencing of more mutations per sample is desired, additional DNA barcodes can be used. Another consideration is that the short length of the reads hinders the detection of large indels or complex mutations (multiple mutations occurring across the same DNA molecule). However, read lengths for the different platforms continue to increase [45] and may eventually increase to a point where complex mutations can be detected with a standard library.

Our method was designed using DNA barcoding to allow for cost- and time-effective analysis of lacZ mutation spectra. We estimate that the cost to sequence 3872 mutants using Sanger sequencing would be 20 -fold higher than using NGS in reagent costs alone. Furthermore, this sequencing can be done in a week using NGS, while it would take at least 25 times as long using Sanger sequencing. If more mutants were sequenced, whether they were from the same samples or different experiments, the difference in cost and time would be greater. As demonstrated, there is an added bonus of sequencing large numbers of mutants: 1) mutation spectra can be characterized with high precision; 2) novel mutational hotspots can be identified (important for understanding an agent's mode of action); and 3) silent mutations present on a mutated transgene, which are normally undetected, can be characterized. Considering that each barcoded library was sequenced with an average coverage of 91,000x (182,000x coverage per sample), several more samples could have been sequenced, making this approach highly efficient and amenable to the simultaneous sequencing of mutant plaques from different tissues and/or chemical agents. Furthermore, the cost and time to do these experiments will decline further as the throughput of NGS increases.

\section{Conclusions}

In summary, we developed a high-throughput method for targeted-sequencing of long mutant reporter genes, specifically the lac $Z$ transgene. The mutation spectra determined using the NGS analytical process are highly concordant with results using Sanger sequencing, demonstrating the accuracy of the methodology. After the animal exposure and tissue sample collection is complete, a whole experiment, from the beginning of the transgenic rodent assay to the end of the data analysis, can be done in less than 3 weeks using our newly established data analysis. The benefit of this method is that it can sequence as many mutants as the TGR assay can produce. Thus, this method has the potential to sequence hundreds to thousands of mutants from different tissues, exposure groups, and doses simultaneously. Despite the apparently low recovery of mutants by NGS due to the reduced sensitivity of calling mutations from pooled DNA, the rapid characterization of large numbers of mutants provides a more accurate measure of mutation spectra and clonal expansion. Although the method is applied to sequencing lac $Z$ mutants, this approach can be modified to identify mutations in any reporter gene used in genetic toxicity testing. With the higher throughput and reduced cost offered by NGS, mutations induced by drugs or chemicals can now be more routinely assessed. Understanding the mode of action of different mutagenic agents enables improved regulation of genotoxins and ultimately a reduction in harmful diseases such as cancer.

\section{Availability of supporting data}

Supporting data are available on the NCBI Sequence Read Archive under the BioProject accession number PRJNA284951.

\section{Additional file}

Additional file 1: Figure S1. Comparison of mutant read proportions for substitutions at each nucleotide position across all samples. Figure S2. Example density graphs (smoothed histograms) for variant calls at two different nucleotide positions. Figure S3. Distribution of read starts and ends. Figure S4. Comparison of the density of false mutation proportions between base substitutions (red) and indels (blue). Figure S5. Relative proportion of control and BaP-induced lacl mutations in liver from reference [29]. Figure S6. Bone marrow mutation spectra of control 
and $\mathrm{BaP}$ treatments. Figure S7. Comparison of spontaneous mutation spectra between our NGS dataset and other published datasets $[4,5,7,9$ 11]. Figure S8. Comparison of BaP-induced mutation spectrum in bone marrow measured using NGS with the mean mutation spectrum measured from four tissues (forestomach, spleen, colon, glandular stomach) using Sanger sequencing [6]. Figure S9. Distribution of non-unique base substitutions across the lacZ transgene for control and BaP samples. Figure S10. Comparison of lacZ mutation hotspots identified using Sanger sequencing [5-10] with mutations identified using NGS at the same nucleotide positions in the present study. Figure S11. Distribution of nonunique indels across the lacZ transgene for control and BaP samples. Figure S12. Results of simulations that use random sampling of BaP mutants to approximate the number of mutants per sample required to achieve a consistent mutation spectrum. Table S1. Thresholds used to detect mutations from each sample library. Table S2. Comparison of expected and observed mutant count when the number of each mutant plaque was controlled for in each NGS library. Table S3. Percent Clonality and Adjusted Mutant Frequencies in Control and BaP-treated samples using the LOD/linear model to adjust mutant counts. Table S4. Comparison of BaP-induced and spontaneous mutation spectra. (DOCX $418 \mathrm{~kb}$ )

\section{Abbreviations}

BaP: Benzo[a]pyrene; NGS: Next-generation sequencing; TGR: Transgenic rodent.

\section{Competing interests}

The authors declare that they have no competing interests.

\section{Authors' contributions}

$M A B, F M$, and $C L Y$ designed the study. MAB and RG performed the experiments and data analysis. AW developed the clonality model. All authors contributed to writing the manuscript and approved the final manuscript.

\section{Acknowledgements}

The authors would like to thank Ahmad Ghadiri, John Gingerich, Lynda Soper, Beverly Allan, Dr. Matthew Meier, and Dr. Jason O'Brien for their contributions to this project. This research was supported by the Canadian Regulatory System for Biotechnology. Stipend support was provided by the Natural Sciences and Engineering Research Council of Canada and the Canadian Institute of Health Research (CIHR) Training Program in Reproduction, Early Development, and the Impact on Health.

\section{Author details}

${ }^{1}$ Carleton University, Ottawa, ON K1S 5B6, Canada. ${ }^{2}$ Environmental Health Science and Research Bureau, Healthy Environments and Consumer Safety Branch, Health Canada, Ottawa, ON K1A OK9, Canada.

\section{Received: 22 February 2015 Accepted: 3 October 2015}

\section{Published online: 19 October 2015}

\section{References}

1. Gossen JA, De Leeuw W, Tan C, Zwarthoff EC, Berends F, Lohman P, et al. Efficient rescue of integrated shuttle vectors from transgenic mice: a model for studying mutations in vivo. Proc Natl Acad Sci. 1989;86(20):7971-5.

2. Lambert IB, Singer TM, Boucher SE, Douglas GR. Detailed review of transgenic rodent mutation assays. Mutat Res Rev Mutat Res. 2005;590(1):1-280.

3. OECD: OECD guideline for the testing of chemicals. Transgenic Rodent Somatic and Germ Cell Gene Mutation Assays. 2011. Doi:10.1787/20745788

4. Vijg J, Douglas G. Bacteriophage lambda and plasmid lacZ transgenic mice for studying mutations in vivo. In: Gerd P, editor. PfeiferTechnologies for Detection of DNA Damage and Mutations. New York: Plenum Press; 1996. p. 391-410. http://link.springer.com/chapter/10.1007/978-1-4899-0301-3_29.

5. Douglas GR, Gingerich JD, Gossen JA, Barlett SA. Sequence spectra of spontaneous lacZ gene mutations in trransgenic mouse somatic and germline tissues. Mutagenesis. 1994;9(5):451-8.

6. Hakura A, Tsutsui Y, Sonoda J, Tsukidate K, Mikami T, Sagami F. Comparison of the mutational spectra of the lacZ transgene in four organs of the Muta ${ }^{\mathrm{TM}}$ Mouse treated with benzo [a] pyrene: target organ specificity. Mutat Res Fundam Mol Mech Mutagen. 2000;447(2):239-47.
7. Ono T, Ikehata H, Nakamura S, Saito Y, Hosoi Y, Takai Y, et al. Age-associated increase of spontaneous mutant frequency and molecular nature of mutation in newborn and old lacZ-transgenic mouse. Mutat Res Fundam Mol Mech Mutagen. 2000;447(2):165-77.

8. Ikehata H, Takatsu M, Saito Y, Ono T. Distribution of spontaneous CpG-associated G: $\mathrm{C} \rightarrow \mathrm{A}:$ T mutations in the lacZ gene of Muta ${ }^{\mathrm{TM}}$ mice: Effects of CpG methylation, the sequence context of CpG sites, and severity of mutations on the activity of the lacZ gene product. Environ Mol Mutagen. 2000;36(4):301-11.

9. Douglas GR, Jiao J, Gingerich JD, Gossen JA, Soper LM. Temporal and molecular characteristics of mutations induced by ethylnitrosourea in germ cells isolated from seminiferous tubules and in spermatozoa of lac $Z$ transgenic mice. Proc Natl Acad Sci. 1995;92(16):7485-9.

10. Douglas GR, Jiao J, Gingerich JD, Soper LM, Gossen JA. System issues: Temporal and molecular characteristics of lacZ mutations in somatic tissues of transgenic mice. Environ Mol Mutagen. 1996;28(4):317-24.

11. Besaratinia A, Li H, Yoon J, Zheng A, Gao H, Tommasi S. A high-throughput next-generation sequencing-based method for detecting the mutational fingerprint of carcinogens. Nucleic Acids Res. 2012;40(15):e116-6.

12. Harismendy O, Ng PC, Strausberg RL, Wang X, Stockwell TB, Beeson KY, et al. Evaluation of next generation sequencing platforms for population targeted sequencing studies. Genome Biol. 2009;10(3):R32.

13. Granville CA, Hanley NM, Mumford JL, DeMarini DM. Mutation spectra of smoky coal combustion emissions in Salmonella reflect the TP53 and KRAS mutations in lung tumors from smoky coal-exposed individuals. Mutat Res Fundam Mol Mech Mutagen. 2003;525(1):77-83.

14. Moriya M, Spiegel S, Fernandes A, Amin S, Liu T, Geacintov N, et al. Fidelity of translesional synthesis past benzo [a] pyrene diol epoxide-2'deoxyguanosine DNA adducts: Marked effects of host cell, sequence context, and chirality. Biochemistry. 1996;35(51):16646-51.

15. Fernandes A, Liu T, Amin S, Geacintov NE, Grollman AP, Moriya M. Mutagenic potential of stereoisomeric bay region (+)-and (-)-cis-anti-benzo [a] pyrene diol epoxide-N 2-2'-deoxyguanosine adducts in Escherichia coli and simian kidney cells. Biochemistry. 1998;37(28):10164-72.

16. Yu D, Berlin JA, Penning TM, Field J. Reactive oxygen species generated by $\mathrm{PAH}$ o-quinones cause change-in-function mutations in p53. Chem Res Toxicol. 2002;15(6):832-42.

17. DeMarini DM, Landi S, Tian D, Hanley NM, Li X, Hu F, et al. Lung tumor KRAS and TP53 mutations in nonsmokers reflect exposure to PAH-rich coal combustion emissions. Cancer Res. 2001;61(18):6679-81.

18. Shwed PS, Crosthwait J, Douglas GR, Seligy VL. Characterisation of MutaTM Mouse $\lambda$ gt10-lacZ transgene: evidence for in vivo rearrangements. Mutagenesis. 2010;25(6):609-16.

19. Gossen J, Molijn A, Douglas G, Vijg J. Application of galactose-sensitive E. coli strains as selective hosts for LacZ-plasmids. Nucleic Acids Res. 1992;20(12):3254

20. O'Brien JM, Beal MA, Gingerich JD, Soper $L$, Douglas GR, Yauk $C L$, Marchetti F. Transgenic rodent assay for quantifying male germ cell mutant frequency. JoVE (Journal of Visualized Experiments). 2014,(90):e51576.

21. Rothberg JM, Hinz W, Rearick TM, Schultz J, Mileski W, Davey M, et al. An integrated semiconductor device enabling non-optical genome sequencing. Nature. 2011;475(7356):348-52.

22. Langmead B, Salzberg SL. Fast gapped-read alignment with Bowtie 2. Nat Methods. 2012;9(4):357-9.

23. Kalnins A, Otto K, Rüther U, Müller-Hill B. Sequence of the lacZ gene of Escherichia coli. EMBO J. 1983;2(4):593.

24. Li H, Handsaker B, Wysoker A, Fennell T, Ruan J, Homer N, et al. The sequence alignment/map format and SAMtools. Bioinformatics. 2009;25(16):2078-9.

25. Team RC. R: a language and environment for statistical computing. Vienna, Austria: R Foundation for Statistical Computing; 2013

26. Chambers JM. Linear models. 1992.

27. Wilkinson G, Rogers C. Symbolic description of factorial models for analysis of variance. Appl Stat. 1973;22:392-99.

28. Lemieux CL, Douglas GR, Gingerich J, Phonethepswath S, Torous DK, Dertinger SD, et al. Simultaneous measurement of benzo [a] pyrene-induced Pig-a and lacZ mutations, micronuclei and dna adducts in mutaTMmouse. Environ Mol Mutagen. 2011;52(9):756-65.

29. Shane BS, de Boer J, Watson DE, Haseman JK, Glickman BW, Tindall KR. Lac mutation spectra following benzo [a] pyrene treatment of Big Blue ${ }^{\circledast}$ mice. Carcinogenesis. 2000;21(4):715-25 
30. Baird WM, Hooven LA, Mahadevan B. Carcinogenic polycyclic aromatic hydrocarbon-DNA adducts and mechanism of action. Environ Mol Mutagen. 2005;45(2-3):106-14

31. Miller KP, Ramos KS. Impact of cellular metabolism on the biological effects of benzo [a] pyrene and related hydrocarbons. Drug Metab Rev. 2001;33(1):1-35.

32. Singh R, Sram RJ, Binkova B, Kalina I, Popov TA, Georgieva T, et al. The relationship between biomarkers of oxidative DNA damage, polycyclic aromatic hydrocarbon DNA adducts, antioxidant status and genetic susceptibility following exposure to environmental air pollution in humans. Mutat Res Fundam Mol Mech Mutagen. 2007;620(1):83-92.

33. Gelboin HV. Benzo[alpha]pyrene metabolism, activation and carcinogenesis: role and regulation of mixed-function oxidases and related enzymes. Physiol Rev. 1980;60(4):1107-66.

34. Wang JJ, Marshall WD, Frazer DG, Law B, Lewis DM. Characterization of DNA adducts from lung tissue of asphalt fume-exposed mice by nanoflow liquid chromatography quadrupole time-of-flight mass spectrometry. Anal Biochem. 2003;322(1):79-88.

35. Thybaud V, Dean S, Nohmi T, de Boer J, Douglas GR, Glickman BW, et al. In vivo transgenic mutation assays. Mutat Res Fundam Mol Mech Mutagen. 2003:540(2):141-51.

36. O'Brien JM, Williams A, Gingerich J, Douglas GR, Marchetti F, Yauk CL. No evidence for transgenerational genomic instability in the F1 or F2 descendants of Muta ${ }^{\text {TM }}$ Mouse males exposed to N-ethyl-N-nitrosourea. Mutat Res Fundam Mol Mech Mutagen. 2013;741-742:11-7.

37. Beal MA, Glenn TC, Somers CM. Whole genome sequencing for quantifying germline mutation frequency in humans and model species: Cautious optimism. Mutat Res Rev Mutat Res. 2012;750(2):96-106.

38. Snyder M, Du J, Gerstein M. Personal genome sequencing: current approaches and challenges. Genes Dev. 2010;24(5):423-31.

39. Koboldt DC, Ding L, Mardis ER, Wilson RK. Challenges of sequencing human genomes. Brief Bioinform. 2010;11(5):484-98.

40. Farrer RA, Henk DA, MacLean D, Studholme DJ, Fisher MC. Using False Discovery Rates to Benchmark SNP-callers in next-generation sequencing projects. Sci Rep. 2013;3:1512.

41. Margulies M, Egholm M, Altman WE, Attiya S, Bader JS, Bemben LA, et al. Genome sequencing in microfabricated high-density picolitre reactors. Nature. 2005;437(7057):376-80.

42. Huse SM, Huber JA, Morrison HG, Sogin ML, Welch DM. Accuracy and quality of massively parallel DNA pyrosequencing. Genome Biol. 2007:8(7):R143

43. Pienaar $\mathrm{E}$, Theron M, Nelson M, Viljoen HJ. A quantitative model of error accumulation during PCR amplification. Comput Biol Chem. 2006;30(2):102-11.

44. Schmitt MW, Kennedy SR, Salk JJ, Fox EJ, Hiatt JB, Loeb LA. Detection of ultra-rare mutations by next-generation sequencing. Proc Natl Acad Sci. 2012;109(36):14508-13.

45. Liu L, Li Y, Li S, Hu N, He Y, Pong R, et al. Comparison of next-generation sequencing systems. J Biomed Biotechnol. 2012;2012:251364.

\section{Submit your next manuscript to BioMed Central and take full advantage of:}

- Convenient online submission

- Thorough peer review

- No space constraints or color figure charges

- Immediate publication on acceptance

- Inclusion in PubMed, CAS, Scopus and Google Scholar

- Research which is freely available for redistribution 